\title{
Optimisation of Dispersion Compensating in a Long-haul Fibre for RF Transmission of up to 100Gbit/s by using RZ and NRZ Formats
}

\author{
Fadil Paloi, Taimur Mirza and *Shyqyri Haxha \\ Department of Computer Science and Technologies, University of Bedfordshire, University \\ Square, Luton, Bedfordshire, LU1 3JU, United Kingdom, *Shyqyri.Haxha@beds.ac.uk
}

\begin{abstract}
With the recent data rate increase it is very challenging to build a fibre optic network that would enable a high data rate transmission over a long haul distance. The signal suffers large degradation over a certain distance due to distortion by the nonlinear effects of the optical fibres. In particular, transmission of high data rates over existing fibre optic systems, while keeping the cost low, avoiding an increase of the system's complexity and the usage of expensive devices, would be a very challenging task. In this paper, we address this problem by increasing the transmission distance in the fibre optic links for up to $2500 \mathrm{~km}$. We have used Standard Single Mode Fibre (SSMF) and Dispersion Compensation Fibre (DCF), where DCF is used as a loss compensator in Radio-Over-Fibre (RoF) systems. A mixture combination of the pre, post and symmetrical fibre compensation schemes were developed to overcome the dispersion in the fibre. We have found that in order to achieve high RF over fibre optic system performance for high data rates and long transmission, there is a requirement to upgrade the optical configuration scheme in a proportional way, by raising the length of the fibre span, compensation span and amplification. We have reported optimised RF over fibre configuration schemes that would have a great impact on reducing the cost, reducing the system's complexity and avoiding usage of expensive devices, in order to achieve high data rate transmission over existing fibre optic systems.
\end{abstract}

Keywords: Fiber optics and optical communications; Fiber optics links and subsystems; Single Mode Fibre.

\section{Introduction.}

In today's era, telecommunication industry has become the backbone of communication around the world, and the developments in the telecommunication sector have brought many wired and wireless technologies [1]. Radio frequency (RF) transmission over optical fibre links is the most reliable and fastest form of communication for long-haul and also for shorter distance transmission systems [1-3]. Within the decade, it is expected to have a large divergence between the growth on traffic demand and created capacity [2]. With the rapid increase in the number of simultaneous multi-users, the communication infrastructure industry is facing significant challenges to transport high bandwidth over longer transmission distances [3]. Considering the nonlinearities and dispersions in the optical fibre, it is almost impossible to transport high bandwidth signals through a long-haul fibre, as it causes an increase in the bit error, which results in irretrievable data at the receiverend. The nonlinear impairments in the Single Mode Fibre (SMF) are mainly caused by chromatic dispersion and polarisation mode dispersion (PMD). Chromatic dispersion is induced by the change in the refractive index, which influences the difference in the group velocity of the wavelengths of a signal [4-6]. High bandwidth transmission is severely 
affected in a single channel transmission by the polarisation mode dispersion and group velocity dispersion (GVD). Thus, pulses at the receiver arrive with delays causing a limitation of the received information, and restricting the number of input users [7, 8]. Large user traffic is being tackled by using transmission techniques like Wavelength Division Multiplexing (WDM), which allows ' $N$ ' number of channels to transmit data through a single fibre. Hence, by optimising and analysing the single channel transmission, a wide understanding of the WDM can be achieved $[9,10]$. To prevent these errors, dispersion needs to be compensated. In the last decade, many techniques have been developed known as all optical, based on the Dispersion Compensating Fibre (DCF). [11, 12].

There are a few dispersion compensation schemes to overcome the dispersion in the fibre, such as pre-compensation, post-compensation and symmetrical compensation schemes [1315]. To increase the transmission distance in the fibre optic links for up to $2500 \mathrm{~km}$, we have used SSMF and DCF. DCF is used as loss compensator in RoF Systems, where for the SMF dispersion is $16 \mathrm{ps} / \mathrm{nm} . \mathrm{km}$ [16]. For the fibre length of $100 \mathrm{~km}$, dispersion of $16 \times 100=1600 \mathrm{ps} / \mathrm{nm}$ is accumulated. To compensate for the accumulated dispersion in the SSMF, a $20 \mathrm{~km}$ long DCF with a negative dispersion of $80 \mathrm{ps} / \mathrm{nm} . \mathrm{km}$ is required $(20 \mathrm{x}-80=$ $-1600 \mathrm{ps} / \mathrm{nm}$ ). Previously, great amount of work has been done on the fibre dispersion compensation by using the pre, post and symmetrical compensation schemes. In [16], the comparison of the pre-, post-, and symmetrical compensation schemes is proposed based on a NRZ format for only 10Gbit/s. The dispersion coefficient used for SMF and DCF is $16 \mathrm{ps} / \mathrm{nm} . \mathrm{km}$ and $-80 \mathrm{ps} / \mathrm{nm} . \mathrm{km}$ respectively. The comparison indicates that the symmetrical compensation scheme has the ability to compensate the fibre impairments with greater efficiency as compared to the other dispersion compensation schemes.

In Ref. [17], the similar compensation schemes are compared for 32 channels by using NRZ and RZ formats. The dispersion coefficients used for SMF and DCF are same as [16]. Several articles like Refs. [16-19], the dispersion parameters are similar to the once that we have used in our proposed simulation models. The results from these previous comparisons were analysed and benchmarked. Other impairments with greater efficiency is chromatic dispersion coefficient which will effect in the size of the DCF reel and result in the effective area and bending losses [20, 21].

The proposed configuration is designed and benchmarked by using two highly smart simulation software, such as Optiwave systems and VPI Photonics. The results obtained from the proposed are sufficiently better than the previously reported results. In the proposed configuration the different amplifier spacing is used such as the amplifier spacing in the periodical loop are different than that of outside the periodical loop. Prior to designing and optimising our proposed system configurations, we have benchmarked our Optiwave systems and VPI Photonics with previously published results in Ref. [16 and 17].

In our proposed RF over fibre optic configuration system, we have shown numerous combinations of the SMF and DCF spans, where the increasing dispersion as correspondence to the increasing fibre length is managed by using a combination of three dispersion compensation schemes. In the proposed system, two slightly different configurations of the system are discussed. The first configuration has the fibre span precompensated, which is followed by in-line symmetrical compensation scheme and then a post-compensation is applied again at the end of the fibre link. The second configuration includes the post-compensated fibre at the beginning of the link and then symmetrical compensation scheme in-line and at the end a post-compensated fibre scheme. Pre compensation is used with only reason to compensate any non-linearity's left from modulation, so this type of compensation it will prevent nonlinearities going through the link to the receiver, where possibility is very high they can be boosted when traveling through the link. The other side post compensation is done at the end of the link so it will compensate left losses and irregularities.

Also the system based on two coding formats Return-to-Zero (RZ) and Non-Return to Zero (NRZ) are discussed and demonstrated. RZ format is more robust against the waveform 
distortion than the NRZ format [7]. The modulation formats are basically an on-off key (OOK) of the pulses at a certain duty cycle [22-24]. For the configuration 1, the simulations were done on the Optiwave system [25], whereas the configuration 2 was implemented and analysed on VPI Photonics simulation software [26]. The transmission link of $120 \mathrm{~km}$ to $2500 \mathrm{~km}$ were designed and analysed for a bit rate of $10 \mathrm{Gbit} / \mathrm{s}$ to $100 \mathrm{Gbit} / \mathrm{s}$.

In this paper, a brief introduction of the optical communication transmission system will be presented. In the second section, the methods employed to study the proposed transmission configurations, such as dispersion compensation schemes, are explained. The third section contains the performance related parameters that show how the results are evaluated. In the fourth section, the proposed schemes are explained. The final section includes performance analysis, where the results are examined and compared between two configurations and modulation formats.

\section{Methodology}

The method used in this section will briefly explain the doctrine used for dispersion compensation with special importance on reducing the chromatic dispersion. The format used for optical modulations is on-off-keying (OOK) intensity modulation, which can be either of NRZ or RZ.

\subsection{Dispersion Compensation Scheme}

Chromatic dispersion, also known as material dispersion, can be successfully compensated by using different methods such as DCF [18], chirped Bragg grating [27] and phase conjugation [28]. In this study we are using DCF to compensate for dispersion and we have upgraded and optimised the RF configuration scheme from basic to more advanced in order to investigate its performance.

There are negative effects during the compensation, specifically nonlinearities [29] such as signal degradation caused from the combined effect of group velocity and accumulated emission noise caused from periodic amplification[30, 31]. The system performance depends on the following factors; fibres input power, the optical system configuration and residual dispersion. Dispersion of the fibre can be modelled by using the Taylor expansion around the centre wavelength $[32,33]$ :

$$
\mathrm{D}(\lambda)=\mathrm{D}\left(\lambda_{0}\right)+\mathrm{D}^{\prime}\left(\lambda_{0}\right)\left(\lambda-\lambda_{0}\right)+\frac{1}{2} \mathrm{D}^{\prime \prime}\left(\lambda_{0}\right)\left(\lambda-\lambda_{0}\right)^{2}+\frac{1}{2} \mathrm{D}^{\prime \prime \prime}\left(\lambda_{0}\right)\left(\lambda-\lambda_{0}\right)^{3}
$$

where, $D$ is dispersion caused in the fibre, $D^{\prime}$ is dispersion slope which is the first derivation of the dispersion, $D^{\prime \prime}$ is dispersion caused from bending curvature, which is second derivation and $D^{\prime \prime \prime}$ the third order one. The first two are usually used in consideration such as dispersion $D$ and dispersion slope $D^{\prime}$, as the other higher order terms from Eq. (1) are too small to be compensated and have not been taken in the consideration $[3,11,23]$.

Residual dispersion occurs due to manufacturing and design issues which leads to inadequate slope matching. The factors which will indicate are of a climate nature, such as heat, capacity, thermal conductivity, soil type, moisture content, and in some places snow cover [34]. So residual dispersion is [35]:

$$
\mathrm{D}_{\mathrm{Res}}=\left(\mathrm{D}_{\mathrm{SMF}} * \mathrm{~L}_{\mathrm{SMF}}\right)+\left(\mathrm{D}_{\mathrm{DCF}} * \mathrm{~L}_{\mathrm{DCF}}\right)
$$

As we can see from Eq. (2), residual dispersion has to be neglected in order to obtain perfect compensation, hence [36]:

$$
0=\left(D_{\mathrm{SMF}} * \mathrm{~L}_{\mathrm{SMF}}\right)+\left(\mathrm{D}_{\mathrm{DCF}} * \mathrm{~L}_{\mathrm{DCF}}\right)
$$

To design the proper length of the compensating fibre we have used the following Eq. [35]: 


$$
\mathrm{D}_{\mathrm{SMF}} * \mathrm{~L}_{\mathrm{SMF}}=-\mathrm{D}_{\mathrm{DCF}} * \mathrm{~L}_{\mathrm{DCF}}
$$

To compensate the accumulated positive dispersion, or as it is known pulse broadening, we have used the DCF, which has high negative dispersion where in our case is taken as -80 $\mathrm{ps} / \mathrm{nm}$ per $\mathrm{km}$. When selecting the DCF, it must have low insertion loss, low polarisation mode dispersion and low nonlinearity [37]. DCF is sort of reel mounted in the terminal office, where its size depends on the chromatic dispersion coefficient. A larger chromatic dispersion coefficient will reduce the size of the reel, resulting with a small effective area and large bending losses [5, 20, 38].

\section{Performance Parameters}

The objective of this research is to minimise the bit error rate (BER). BER is a key performance criterion which evaluates the performance of the proposed system. The BER can be expressed in terms of dimensionless parameter such as Q-factor, which is defined as a ratio between the difference of the optical signal intensity and the sum of standard deviation of the optical link at the higher level "1" and lower level "0"[39].

\subsection{Quality Factor (Q-Factor)}

When we discuss analogue transmission, the Q-Factor shows the signal's physical quality strength in terms of SNR. In order to achieve lower probability of bit errors for a given RF over optical transmission system, we must achieve high Q-Factor which means a decent SNR. For the digital signal, Q-Factor represents the quality of the SNR in the eye diagram. If we compare the signals to a reference value $\mathrm{V}_{\text {th }}$ (threshold), when the signal is higher than the threshold it will be indicated as binary "1", whereas if the signal is lower than the threshold it will be indicated as binary "0".

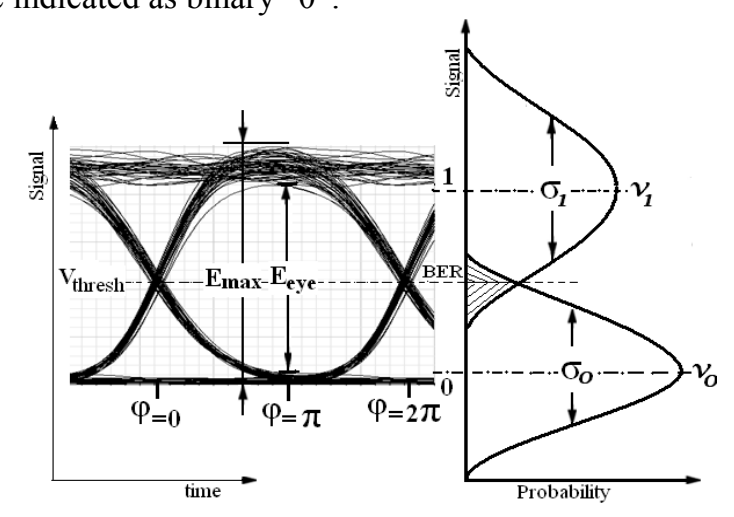

Fig.1: Description of the Signal and Noise with Eye diagram

Performance of the system can be seen by the way the eye diagram is opened " $\mathrm{E}_{\text {eye }}$ ". The larger and clearer the eye opens " $\mathrm{E}_{\text {eye }}$ ", the narrower the distribution signal is, over- lapping is smaller, and Q-Factor is higher [40].

If difference between the mean values of the " 1 " and " 0 ", $\left(\mathrm{v}_{1}\right.$ and $\left.\mathrm{v}_{0}\right)$ is smaller, then distribution noise $\left(\sigma_{1}\right.$ and $\left.\sigma_{0}\right)$ is thicker, overlapping is bigger, Q-Factor is smaller Eq.4.

$$
\mathrm{Q}=\frac{\left|\mathrm{v}_{1}-\mathrm{v}_{0}\right|}{\sigma_{1}+\sigma_{0}}
$$

where $\mathrm{v}_{1}$ and $\mathrm{v}_{0}$ - are related to the level of the transmitted signal data of " 1 " and " 0 " and $\sigma_{1}$ and $\sigma_{0}$ - are related to the degradation of the signal on the " 1 " and " 0 ".

Because the minimum BER occurs at the optimum threshold voltage, BER is related to the Q-Factor and can be calculated by Eq. [39]. 


$$
\operatorname{BER}=\frac{1}{2} \operatorname{erfc}\left(\frac{\mathrm{Q}}{\sqrt{2}}\right) \approx \frac{\exp \left(-\mathrm{Q}^{2} / 2\right)}{\mathrm{Q} \sqrt{2 \pi}}
$$

Eq. (5) is used in general as an approximation of the BER, where "erfe" is error function.

\section{Proposed RF over optical configuration system}

Commercially, a bit rate of up to 10Gbit/s is being used in the fibre optic systems, and the investigation for a higher bit rate of 100Gbit/s and more, over a long-haul fibre is being carried out $[41,42]$. In our study, a configuration of a single channel system for a fibre length of $120 \mathrm{~km}$ to $2400 \mathrm{~km}$, with a bit rate of $10 \mathrm{Gbit} / \mathrm{s}$ to $100 \mathrm{Gbit} / \mathrm{s}$ has been proposed. The system has been optimised for the highest Q-Factor and lowest possible BER by adjusting the power of the optical source and the power of the fibre input and fibre output. The system configuration is illustrated in Fig. 3. In the transmitter, a carrier wave of $1550 \mathrm{~nm}$ wavelength is generated by using a Continuous Wave (CW) laser with a line width of $0.1 \mathrm{MHz}$. The $\mathrm{CW}$ parameters are determined by its power, wavelength, phase, and relative intensity noise (RIN) and line width. CW laser emits constant optical power, which passes through an optical modulator (external), which is a voltage driven device. By adjusting the voltage, absorbed amount of power will vary, and then we will achieve modulation of the optical power.

The RF input signal pulses are generated by using a Pseudo Random Sequence Generator with a NRZ and RZ modulation format. The RZ coding scheme is applied with a duty cycle of 0.25 , which means a ratio of a time of full width of the initial pulses at half maximum (TFWHM) to the bit period $\left(\mathrm{T}_{\mathrm{B}}\right)$.

These ratios and optical signals are modulated by using a Mach-Zehnder external modulator (MZM). The modulated signals are inserted into the optical link, which consists of the transmission fibre spans of $25 \mathrm{~km}$ to $150 \mathrm{~km}$ with attenuation $0.2 \mathrm{~dB} / \mathrm{km}$ and dispersion of 16 $\mathrm{ps} /(\mathrm{nm} . \mathrm{km})$. The Dispersion Compensation Fibre (DCF) is of ranging $5 \mathrm{~km}$ to $30 \mathrm{~km}$, with attenuation of $0.5 \mathrm{~dB} / \mathrm{km}$ and dispersion of $-80 \mathrm{ps} /(\mathrm{nm} . \mathrm{km})$.

The SMF and DCF length depends on the total transmission length of the optical link. For instance, a total transmission length of $120 \mathrm{~km}$ includes four spans of $25 \mathrm{~km} \mathrm{SSMF}$ and four $5 \mathrm{~km}$ DCF spans. To achieve high Q-Factor and low BER we have used four simulated fibre schemes with different combinations of the transmission fibre lengths.

The input power level of each fibre link is adjusted according to the transmission length and bitrate. Erbium-Doped Fibre Amplifier (EDFA) with a noise figure of $6 \mathrm{~dB}$ is used as in a line amplifier, manufactured from Erbium-Doped material composition [43], which is a conventional silica fibre doped with Erbium, operating in the region of $1450 \mathrm{~nm}$ and works perfectly well for the length of $1550 \mathrm{~nm}$ wavelength. These types of amplifiers are used in the WDM systems and have helped to upgrade the field of the fibre communications system.

When the SMF is designed/manufactured, the main challenge is to create fibre with the lowest possible attenuation, the least possible compensation, and building the smallest possible dispersion compensating fibre module (reel), this is because of its size and space which is necessary for it, and ultimately for its cost [11]. The signal suffers large degradation due to distortion by the nonlinear effects of the optical fibres. To address these problems, in the current study we developed a mixture combination of the pre, post and symmetrical fibre compensation [13-17].

This proposed optical configuration scheme will provide negative dispersion before and after the fibre, and eliminate positive dispersion of the SMF. The proper separate use of all three compensations it will lead to higher system performance. The proposed system configuration consists of SMF span of lengths $25 \mathrm{~km}, 50 \mathrm{~km}, 100 \mathrm{~km}$, up to $150 \mathrm{~km}$. The system's performance on these fibre spans are discussed in details below. Outside loop fibre 
length is always higher than in loop, and outside loop amplification boosts amplification inside the loop.

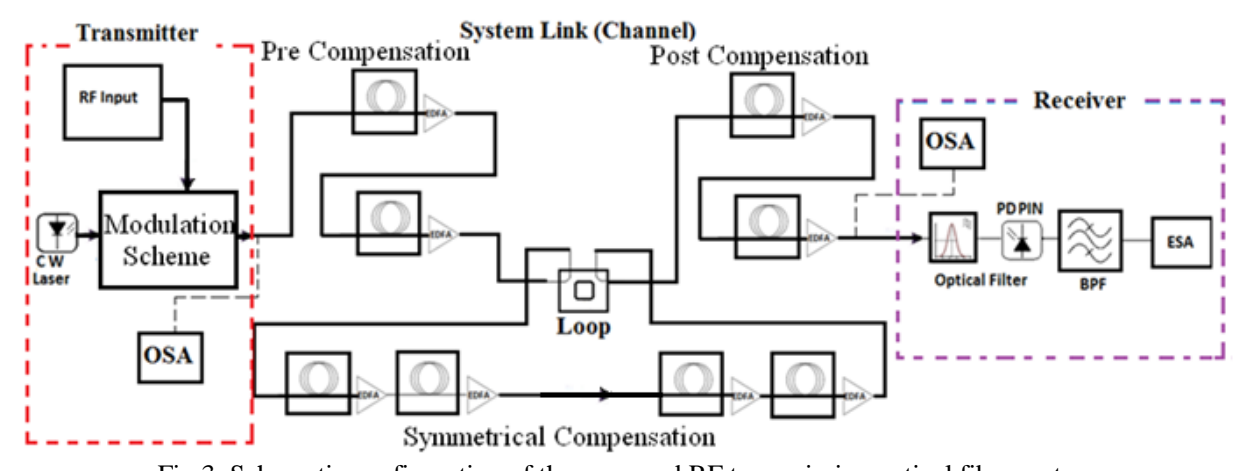

Fig.3. Schematic configuration of the proposed RF transmission optical fibre system

Each span has adequate DCF in order to fully compensate accumulated dispersion and dispersion slope in the transmission link. The losses accumulated by the SMF will be compensated by EDFA gain, using the following expression:

$$
\mathrm{G}=\alpha * \mathrm{~L}
$$

Where $\mathrm{G}$ is Gain (Amplification), $\alpha$ is attenuation (losses) and $\mathrm{L}$ is the fibre length.

During the intensive simulations and after analysing all the results obtained from the proposed optical configuration system it is observed that, in order to achieve a high system performance such as high Q-Factor and low BER, we have to adjust the Gain of amplification outside the loop by $2 / 3$ of the normal Gain. Consequently the following equation is employed:

$$
\mathrm{G}_{\mathrm{out}}=\frac{2}{3} \alpha * \mathrm{~L}
$$

And inside the loop for $10 \%$ less.

$$
\mathrm{G}_{\mathrm{in}}=0.9 \alpha * \mathrm{~L}
$$

In the case of the compensation gain, we have to keep in mind that total Gain of the compensated fibre $\mathrm{G}_{\mathrm{T}_{\mathrm{com}}}$ is sum of Gain of Single Mode Compensated Fibre and Gain of Compensated Fibre:

$$
\mathrm{G}_{\mathrm{T}_{\mathrm{com}}}=\mathrm{G}_{\mathrm{SMc}}+\mathrm{G}_{\mathrm{com}}=\alpha_{\mathrm{SMF}} * \mathrm{~L}_{\mathrm{com}}+\alpha_{\mathrm{com}} * \mathrm{~L}_{\mathrm{com}}
$$

Where $\mathrm{G}_{\mathrm{T}_{\text {com }}}$-is the total gain of compensated fibre, $\mathrm{G}_{\text {com }}$ is the gain for the compensated fibre, and $\mathrm{G}_{\mathrm{SMc}}$ is the gain for the SMF length of compensated fibre.

\section{Performance Analysis}

In this proposed RF over optical system configuration model we are focused on optimising and compensating the dispersion in the fibre link, for the highest possible bitrate and for the longest possible distance, to achieve highest Q-Factor with the lowest BER by comparing the RZ and NRZ coding formats? Simulation results have been analysed and illustrated in order to compare the system's performance for optimised key performance parameters. The presented graphs illustrate the achieved results of Q-Factor and BER. A standard normal BER is specified as $10^{-9}$, which corresponds to the Q-Factor of 5.8 and anything less than Q-Factor equal to 5.8 makes the system less reliable [48]. It is evident that the Q-Factor of more than 41 has zero BER, which makes the system resolute.

In this section we investigate performance of the proposed configuration Fig.3, for the RZ line coding and reference frequency of $1550 \mathrm{~nm}$. Figure 4 , shows Q-Factor in function of 
various transmission distances, using different bitrates. We begin with a bitrate of $10 \mathrm{Gbit} / \mathrm{s}$, where for the length of $120 \mathrm{~km}$ and the lunched power of $20 \mathrm{~mW}$, we could achieve peak QFactor $=198.45$, decreasing to Q-Factor $=116.8$ for the same bitrate and link distance of $240 \mathrm{~km}$. Whereas for the same bitrate $10 \mathrm{Gbit} / \mathrm{s}$, and input power of $10 \mathrm{~mW}$ in one of the cases for the length of $2400 \mathrm{~km}$, a solid Q-Factor=6.3 is achieved. From all calculations and measurements illustrated in Fig 4, Q-Factor penalties originate from nonlinearities as functions of link distances $120 \mathrm{~km}, 240 \mathrm{~km}$, and $1200 \mathrm{~km}$, up to $2400 \mathrm{~km}$, obtained at different bit rate, where the ASE becomes the dominant impairment.

Likewise, we observe peak Q-Factor as function of bitrate in Fig.5, such as 20Gbit/s, 40Gbit/s, 60Gbit/s, 80Gbit/s and 100Gbit/s, where maximum achieved distance can be $2040 \mathrm{~km}, 1440 \mathrm{~km}, 1200 \mathrm{~km}, 960 \mathrm{~km}$ and $840 \mathrm{~km}$ respectively. Fig. 5 as illustrated is expanded to the small axis values so that the achievable Q-Factors can be seen at the margin line of BER $10^{-9}$.

We have carried out similar calculation and simulations for NRZ line coding, where for bitrate of $10 \mathrm{Gbit} / \mathrm{s}$ is achieved Q-Factor $=6.07$, for length of $2160 \mathrm{~km}$. Similarly, for bitrates of $20 \mathrm{Gbit} / \mathrm{s}, 40 \mathrm{Gbit} / \mathrm{s}, 60 \mathrm{Gbit} / \mathrm{s}, 80 \mathrm{Gbit} / \mathrm{s}$, and $100 \mathrm{Gbit} / \mathrm{s}$ the maximum reliable transmission distance is $1320 \mathrm{~km}, 960 \mathrm{~km}, 720 \mathrm{~km}, 660 \mathrm{~km}$ and $480 \mathrm{~km}$ respectively, as illustrated in Fig.6. Q-Factor is in the function of bitrate, depending on different transmission distance. Rapid Q-Factor decline is seen by increasing the link distance, where related Q-Factor penalties are shown in Fig.7.

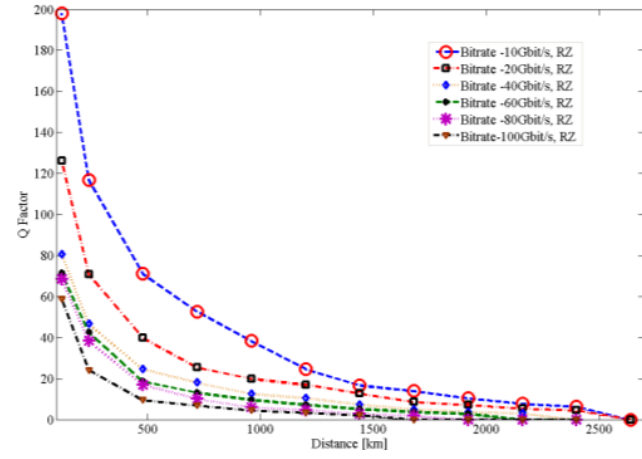

Fig.4: Q-Factor in function of link distance for different bitrates, RZ

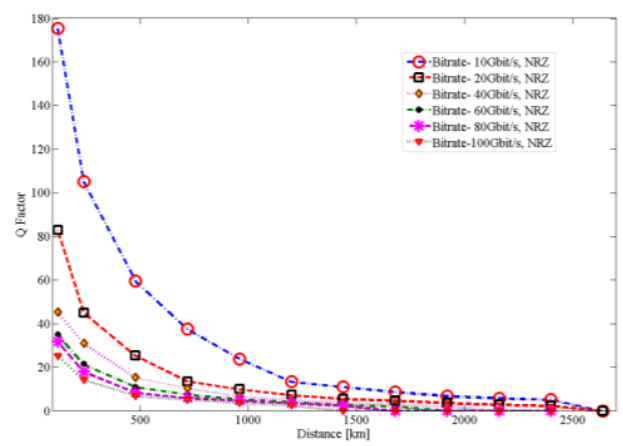

Fig.6: Q-Factor in function of link distance for different bitrates, NRZ.

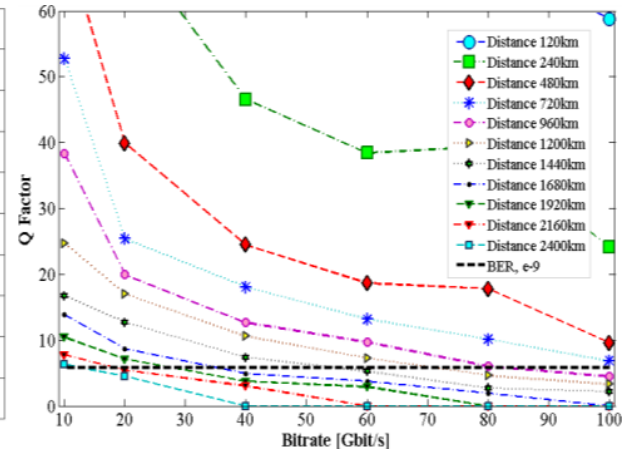

Fig.5: Q-Factor in function of bitrates for RZ and various transmission distances

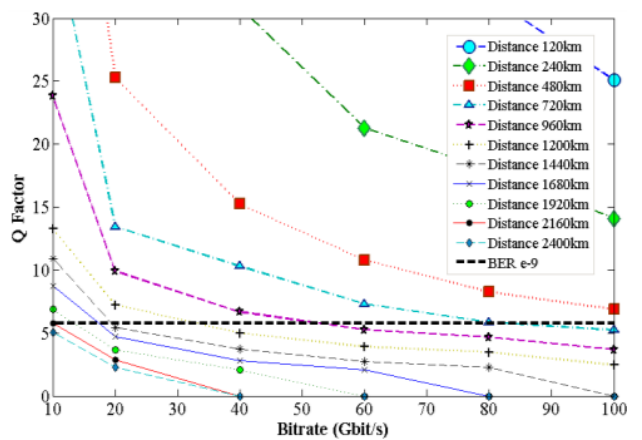

Fig.7: Q-Factor in function of bitrates for NRZ and various transmission distances.

For the long haul transmission Q-Factor penalties are evident because of the additive and multiplicative noise component added to the signal called amplified spontaneous emission (ASE) [31]. Every time we amplify the signal, EDFA inserts to the noise power spectral density (PSD) [30]. 
To analyse the nature of the line coding schemes such as NRZ and RZ, based on the result achieved and discussed we can state that RZ is advancing in the distance compared to NRZ Fig. 5 and Fig. 7, where for $80 \mathrm{Gbit} / \mathrm{s}$, RZ modulation format we can go up to $960 \mathrm{~km}$ and for the same bitrate of NRZ, maximum length achieved is up to $720 \mathrm{~km}$. This can be seen more clearly from the eye diagrams achieved for the link distance of $2400 \mathrm{~km}$ for the RZ and for the link distance of $2160 \mathrm{~km}$ for the NRZ modulating format, Fig. $(8,9)$

From Fig. (5 and 7), we can state that both formats have extremely high quality factor for low bitrates and low length, so they can be considered as ideal, but when bitrate increases, as is apparent from Fig. $(10,11)$, we have rapid decrease on both modulation formats.

Based on our simulation and as shown from results achieved from both modulation formats Fig. (10,11), we have found out that NRZ format is not as appropriate compare to RZ for high data rate and long-haul optical link. RZ format is relatively insensitive to fibre nonlinearities and polarisation mode dispersion compared to NRZ [32].

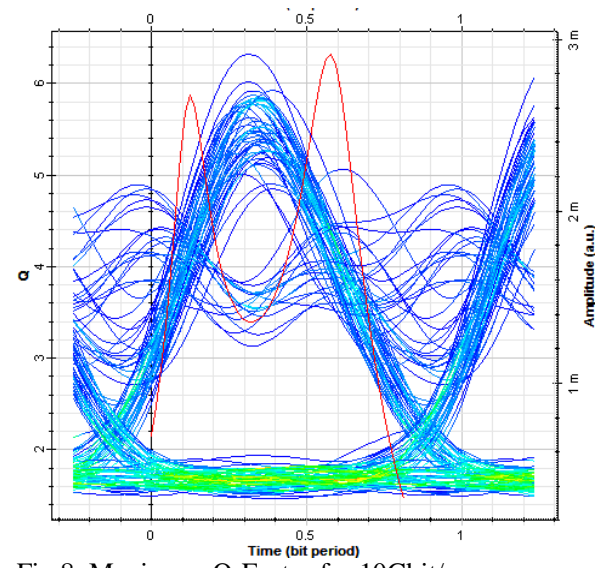

Fig.8: Maximum Q-Factor for 10Gbit/s, $\mathrm{RZ}$, distance of $2400 \mathrm{~km}$

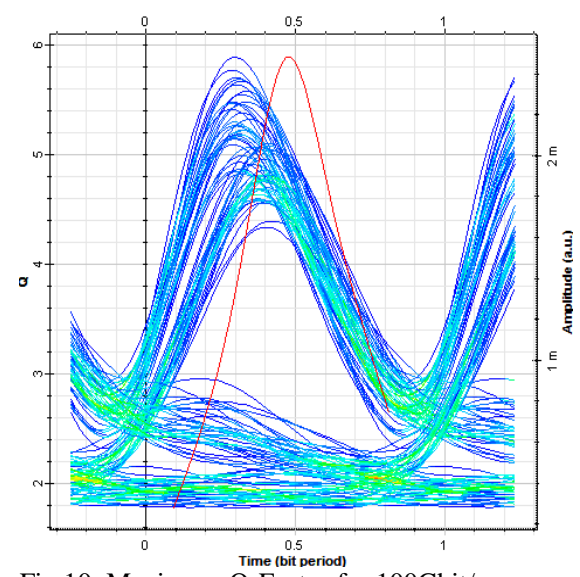

Fig.10: Maximum Q-Factor for 100Gbit/s, $\mathrm{RZ}$, distance of $840 \mathrm{~km}$.

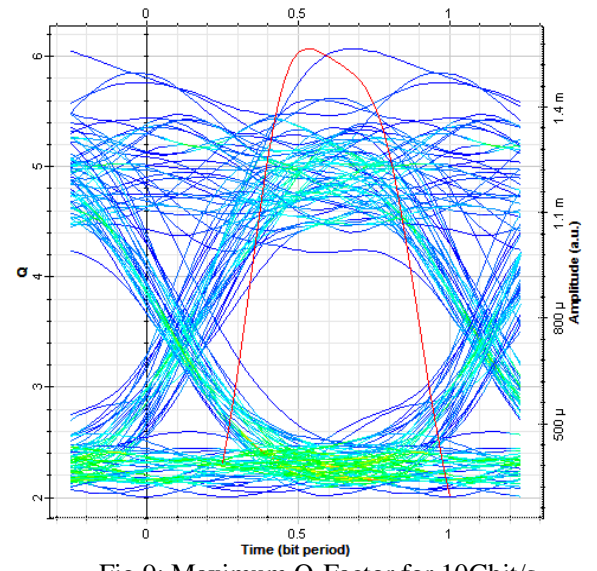

Fig.9: Maximum Q-Factor for 10Gbit/s, NRZ, distance of $2160 \mathrm{~km}$.

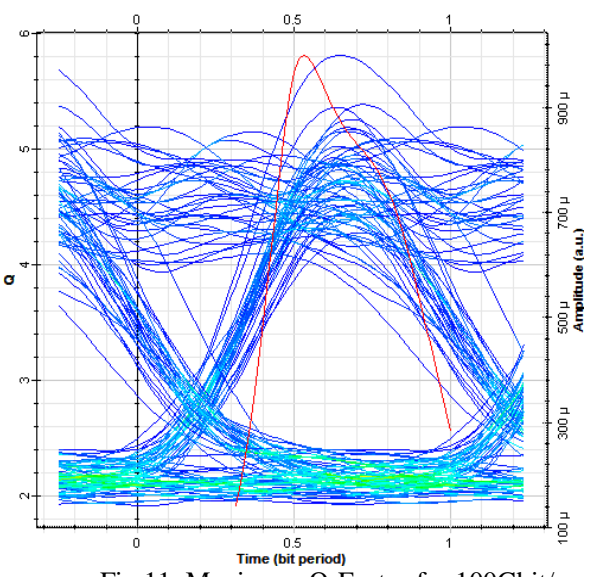

Fig.11: Maximum Q-Factor for 100Gbit/s, NRZ, distance of $480 \mathrm{~km}$.

RZ modulation format is less disposed to the inter-symbol-interference (ISI) as we have seen it achieves much better performance. Other benefits of RZ are self synchronisation, and the extension of lasers life time. With its large bandwidth, RZ modulation format is not well suited with DWDM, where it can be pronounced to other errors such as Intra Four Way Mixing (IFWM), and Intra Cross Phase Modulation [35]. However, the output pulse level is dependent on nonlinearities such as Self Phase Modulation which is the main phenomenon that limits the performance of the single channel light wave system [13].NRZ modulating format has been used extensively because of its easy way of generating and its 
small bandwidth which is around $50 \%$ or more smaller than RZ depending on duty cycles. Throughout all our calculations, we have found out that the use of the same fibre length combination will not give us good results such as Q-Factor and the lowest as possible BER. Because of this, we have used a different combination of the compensation and amplification in the scheme for different input power.

As we have taken an example of 20Gbit/s, and distance of $240 \mathrm{~km}$, RZ has high peak power which is $25[\mathrm{~mW}$ ] compare to $5[\mathrm{~mW}$ ] for NRZ, Fig.12a, which means higher SNR, and very high Q-Factor and lower BER than NRZ modulation encoding format.
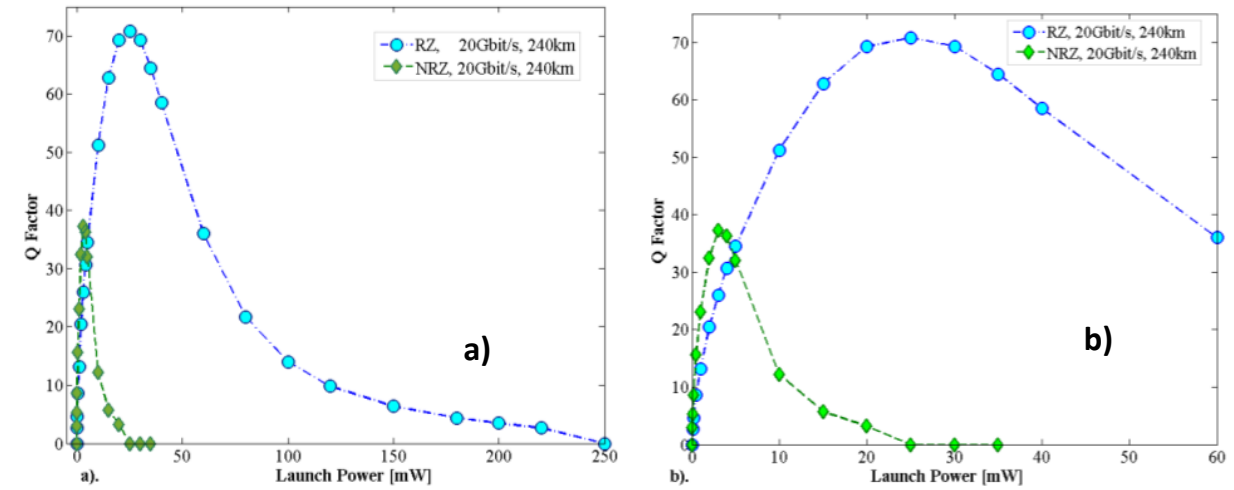

Fig.12: a) Maximum Q-Factor in function of launch power, RZ, NRZ, $20 \mathrm{~GB} / \mathrm{s}, 200 \mathrm{~km}$, b) Expanded view of the Fig.10 (a).

Simulated optical eye margin for different bitrate and for different length of transmission as function of launched power, using the combined compensation are shown in the following section. We observe the eye diagram of the received signal for each bitrate, different length and different launched power. To achieve the highest Q-Factor for each bitrate starting from $10 \mathrm{Gbit} / \mathrm{s}$ up to $100 \mathrm{Gbit} / \mathrm{s}$, we had to upgrade the scheme in a proportional way, by raising length of the fibre span, compensation span and amplification in the proportional way by using Eq. (4, 6, 7, and 8). The fibre length, DCF length and amplification must be regulated on the cascade way inside the loop (mixture compensation).

In Fig.13, we have shown power usage for 10Gbit/s system link and different lengths. As it can be seen peak value of Q-Factor $=198.45$ has been achieved for the length of $120 \mathrm{~km}$ at the launched power of $20 \mathrm{~mW}$. Next we raise the link distance to $240 \mathrm{~km}$, peak value of the Q-Factor decreases to 116.87 , for the launched power of $15 \mathrm{~mW}$. By observing all the data achieved from all calculations and measurements, to achieve high Q-Factor, and by raising the link distance, lunched power decreases too. However, by changing the bitrates to $20 \mathrm{Gbit} / \mathrm{s}$, peak value of Q-Factor has decreased to the value of 126, for the link distance of $120 \mathrm{~km}$ at the lunched power of $20 \mathrm{~mW}$. By raising the distance of the link for the bitrate of 20Gbit/s, Fig.14, the measured Q-Factor was decreased from 126 to 60 for the length of $240 \mathrm{~km}$. However, by increasing the distance, Q-Factor decreases too, and becomes much more constant, i.e. for the length of $1200 \mathrm{~km}$; the Q-Factor is around 20 for almost any launched power Fig.14. This result clearly indicates that the increase of the distance introduces Q-Factor penalty despite the reduction of the amplifiers noise, achieved by using less amplification and less fibre spans. Here, we have noted that using longer distance, QFactor decreases, yet the peak launched power also decreases after a certain distance. By raising the power, we raise the length of the fibre span in a proportional way and that up to $150 \mathrm{~km}$ per span. We have found out, by raising the bitrate the number of fibre spans should be less and length of the fibre span has to be longer. So for low input power and short link length we had to use short fibre span, Fig16.

We found that to achieve similar Q-Factor for $100 \mathrm{Gbit} / \mathrm{s}$ with $480 \mathrm{~km}$ length for RZ modulating format Fig.18, we have to use four different types of cascade optical amplifications and lengths resulting with different input powers. 
For example, if the fibre span in-loop and out-loop are $25 \mathrm{~km}$, the laser power is very low, i.e. $3 \mathrm{~mW}$. If the span out-loop is $50 \mathrm{~km}$ and in-loop is $25 \mathrm{~km}$, the input power goes up to $10 \mathrm{~mW}$ and in the case of fibre span $50 \mathrm{~km}$ out-loop and in-loop increases the input power to $25 \mathrm{~mW}$. With the increase in fibre span length to $100 \mathrm{~km}$ out-loops and $100 \mathrm{~km}$ in-loop, input power goes to $55 \mathrm{~mW}$ as shown in the Fig. 18.

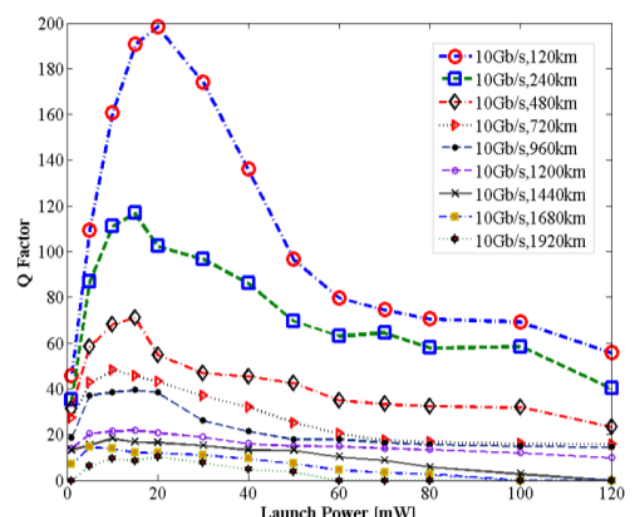

Fig.13: Q-Factor in function of launched power, RZ, $10 \mathrm{Gbit} / \mathrm{s}$, different length.

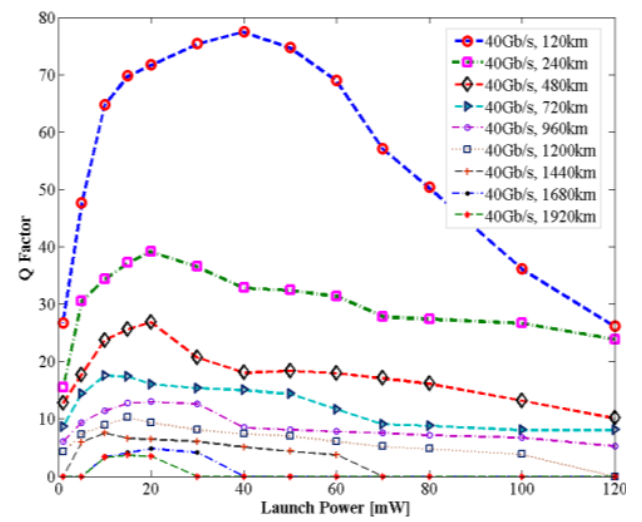

Fig.15: Q-Factor in function of lunched power, RZ, $40 \mathrm{Gbit} / \mathrm{s}$, different length.

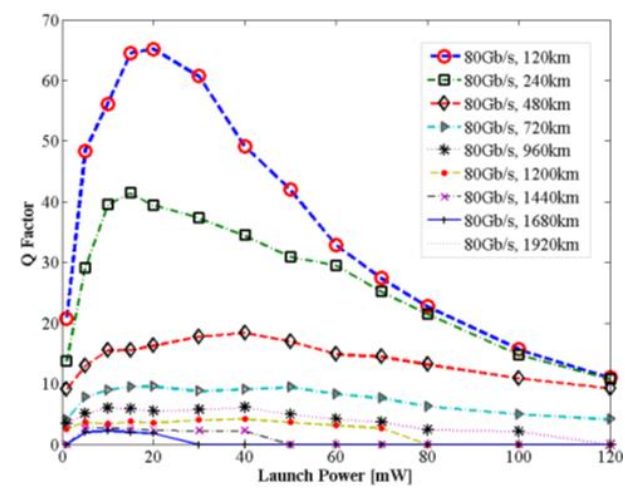

Fig.17: Q-Factor in function of lunched power, RZ, $80 \mathrm{Gbit} / \mathrm{s}$, different length.

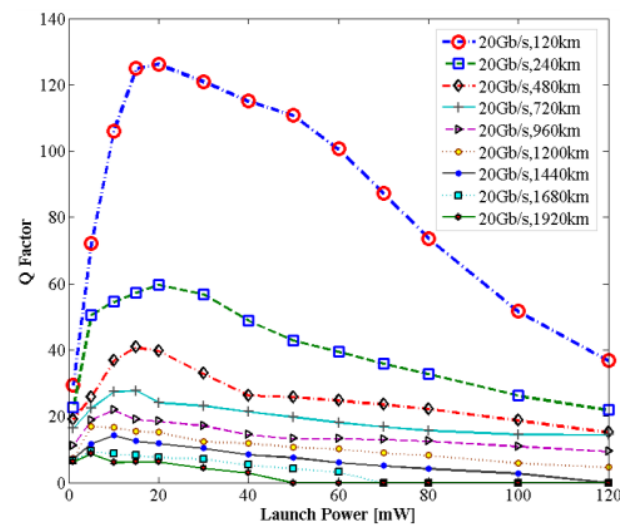

Fig.14: Q-Factor in function of launched, power, RZ, $20 \mathrm{Gbit} / \mathrm{s}$, different length.

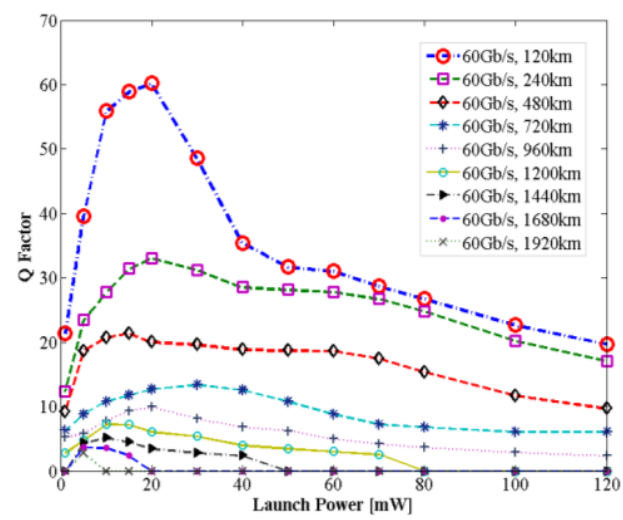

Fig.16: Q-Factor in function of lunched power, RZ, $60 \mathrm{Gbit} / \mathrm{s}$, different length.

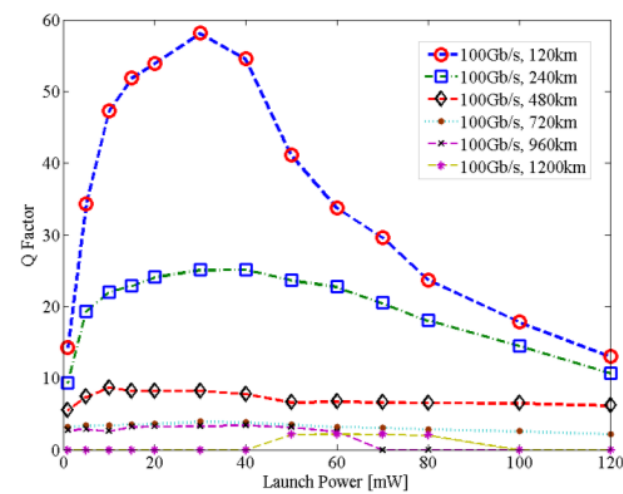

Fig.18: Q-Factor in function of lunched power, RZ, $100 \mathrm{Gbit} / \mathrm{s}$, different length.

Similarly, for the NRZ modulating format to achieve similar Q Factor, for100 Gbit/s with $480 \mathrm{~km}$ length, for the fibre span in-loop and out-loop $25 \mathrm{~km}$, the input power has to be very low $0.5 \mathrm{~mW}$. If the span out-loops go up to $50 \mathrm{~km}$ and the in-loop is $25 \mathrm{~km}$, the input power goes up to $1.5 \mathrm{~mW}$ and in the case of fibre span $50 \mathrm{~km}$ out-loop and in-loop increases the 
input power to $3 \mathrm{~mW}$. With the increase in fibre span length to $100 \mathrm{~km}$ out-loops and $50 \mathrm{~km}$ in-loop, input power goes up to $7 \mathrm{~mW}$.

\section{Comparison of both proposed configuration schemes}

In the figures below, the comparison of the two proposed configurations of the systems based on RZ and NRZ modulation formats are shown. Configuration 1 in the graphs represents the compensation scheme based on the pre-compensation, symmetrical and post compensation method, which is simulated by the Optiwave design software for photonics, whereas, configuration 2 is based on the post, symmetrical and post techniques for the dispersion compensation, that is simulated by the VPI photonics software.

In Fig. 19, the transmission for a data rate of 10Gbit/s is evaluated, and both configured systems for RZ and NRZ have achieved extremely high Q-Factor. Configuration 1 for both modulation formats appears to be more robust against the losses as compared to configuration 2. This indicates to us that regardless of the coding formats, the first configuration gives better results for the low data rate and also performs well overall. Both formats for the first configuration have very good Q-Factor that can potentially reach the maximum transmission length of $2400 \mathrm{~km}$. We can say this comes because, pre compensation has compensate non-linearity's left from modulation and has prevent nonlinearities getting boosted when traveling through the link to the receiver. The other side post compensation has done its job by compensating left losses and irregularities before the receiver.

Both configurations perform almost the same for the transmission length of up to $960 \mathrm{~km}$ at a data Rate of 40Gbit/s, and then after this length both curves start to fall apart as shown in Fig. 21. The first configuration still has the capacity of carrying data to over $1680 \mathrm{~km}$, whereas the second configuration can only manage to reach to a distance of around $1200 \mathrm{~km}$. For NRZ format, the first configuration can transmit data over a maximum length of approximately $1080 \mathrm{~km}$ with a reliable Q-Factor of 6.66 , but the second configuration has a capacity to reach a transmission length of up to $720 \mathrm{~km}$. With the transmission size of $60 \mathrm{Gbit} / \mathrm{s}$ Fig. 22, configuration 1 can potentially reach a maximum distance of $1320 \mathrm{~km}$ and configuration 2 can transmit over $1080 \mathrm{~km}$ long transmission link. The results lower than Q factors of 6 are full of noise and impairments. Fig.23 depicts the results of using 80Gbit/s for both system configurations, where the first configuration with a RZ modulation can carry out a reliable transmission over a distance of $960 \mathrm{~km}$. Whereas, for NRZ modulation format can carry out transmission over a distance of $720 \mathrm{~km}$.

By looking at the graph Fig.24, the behaviour of the curves can be analysed. The constant decline in the quality factor with the increase in transmission length and data rate is noticed. For the transmission of $100 \mathrm{Gbit} / \mathrm{s}$ data, only a maximum length of $840 \mathrm{~km}$ can be achieved, which is only by using configuration 1 with RZ format. However, configuration 2 is unstable for this transmission and only has the capacity to transmit over a distance of $360 \mathrm{~km}$. RZ format, because of the short pulse which occupies a broader bandwidth and the high bitrate as compared to NRZ signal, will suffer more from the distortion caused by the combination of higher order GVD and SPM.

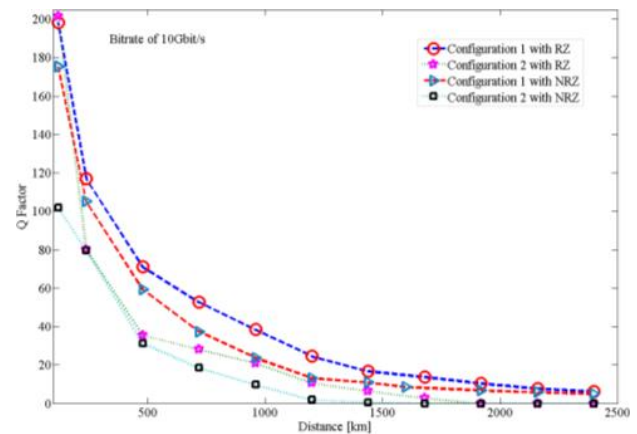

Fig.19: Q-Factor in function of distance, $10 \mathrm{Gbit} / \mathrm{s}(\mathrm{NRZ}$, and RZ).

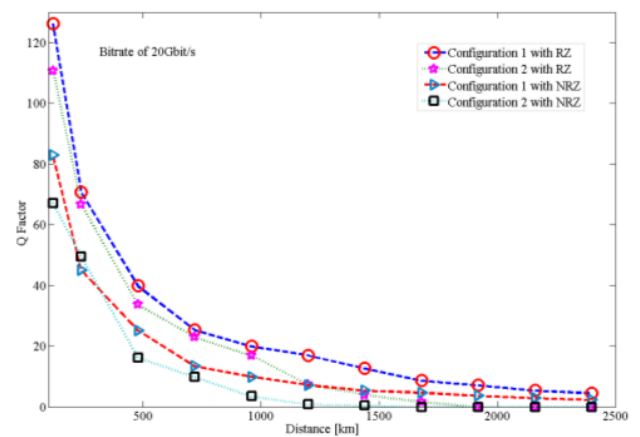

Fig.20: Q-Factor in function of distance, $20 \mathrm{Gbit} / \mathrm{s}$, (NRZ, and RZ). 

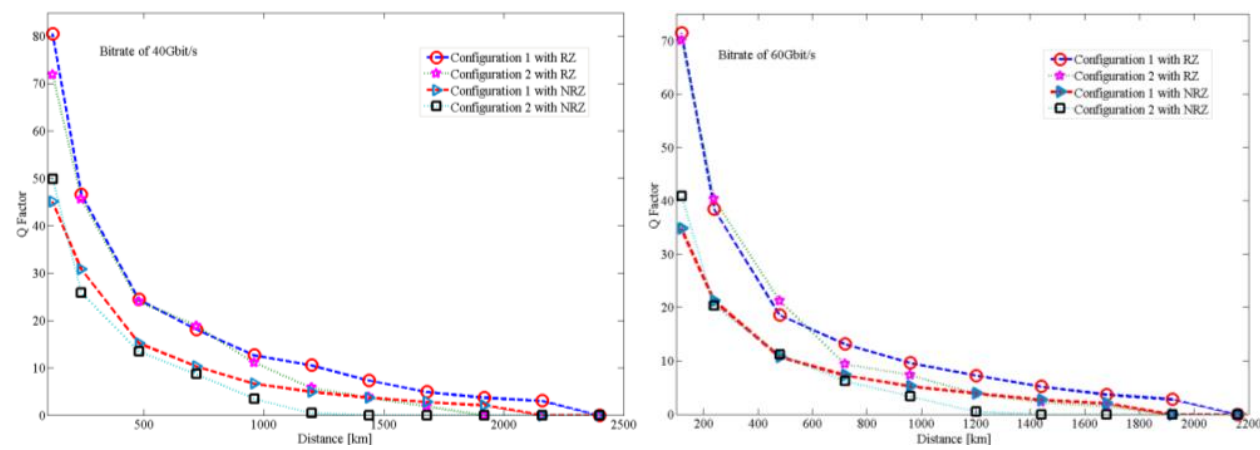

Fig.21: Q-Factor in function of distance, $40 \mathrm{Gbit} / \mathrm{s}$, (NRZ and RZ).

Fig.22: Q-Factor in function of distance, $60 \mathrm{Gbit} / \mathrm{s}$, (NRZ, and RZ).
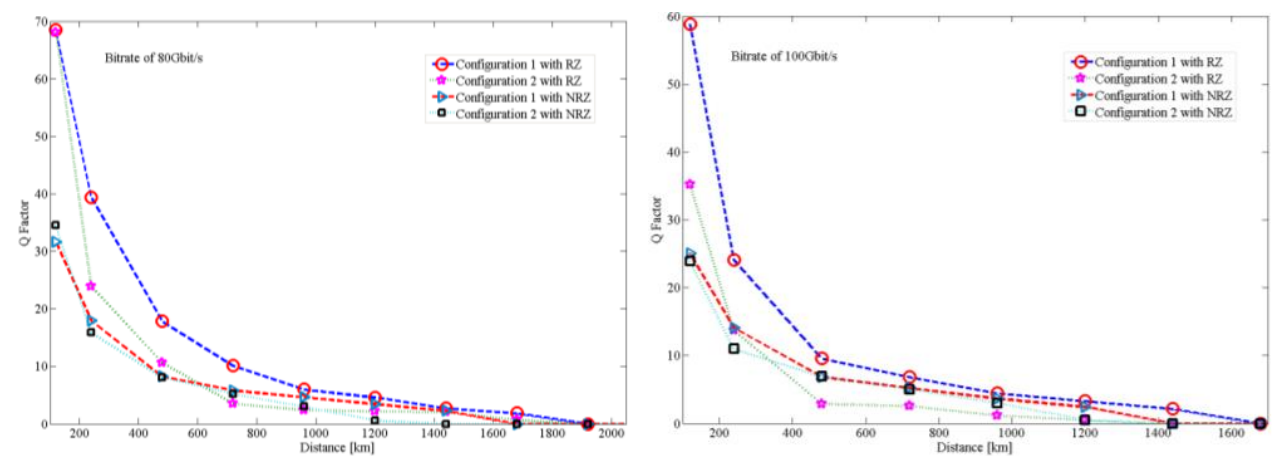

Fig.23: Q-Factor in function of distance,

Fig.24: Q-Factor in function of distance, $80 \mathrm{Gbit} / \mathrm{s}$, (NRZ and RZ).

From the proposed scheme, because of the raise in the bitrate, some of the short length periodical spans will not work. For example, for the modulation format of NRZ, $80 \mathrm{~GB} / \mathrm{s}$, $960 \mathrm{~km}$, we can only use higher than a $50 \mathrm{~km}$ span, the use of $25 \mathrm{~km}$ span length will cause much more losses, where achieved Q-Factor value is lower than the margin line of BER10${ }^{9}$, because of this, and after a certain distance, it is necessary to amplify the signal. During each time signal is amplified, OSNR degrades because the noise is added to the signal, which is called amplified spontaneous emission (ASE) [31]. Each EDFA inserts to the noise power spectral density (PSD) due to the generation of more ASE [30]. When several EDFA are connected along the fibre link, accumulated ASE will weaken the OSNR. When ASE gets higher, amplifiers will get saturated and it will limit amplification of the signal [40].

\section{Conclusion}

We have demonstrated theoretically and by simulation, using Optisystem and VPI, by carrying out numerous RF over fibre optic configuration simulations on the combinations of the SMF, DCF spans and EDFA. We have investigated the achievability of NRZ and RZ coding format for dispersion compensation fibre in the long haul transmission, where we have boost the signal and extend the length of the link for the existing infrastructure with less complexity and low in cost. The proposed RF over fibre optic communication architecture demonstrates that the maximum transmission distance of up to $2400 \mathrm{~km}$ is achieved for a bitrate of $10 \mathrm{Gbit} / \mathrm{s}$ and a distance of around $840 \mathrm{~km}$ for a bit rate of $100 \mathrm{Gbit} / \mathrm{s}$, by using a suitable combination of three dispersion compensation schemes such as pre, post and mixed compensation. It has been demonstrated that optimised appropriate method of the compensation such as the one used in the first configuration can lead to good link performance. It has been demonstrated that the fibre spans for different transmission distances vary, where for a short transmission link, we had to use short fibre spans with low 
launch power. We have shown that the amplifier gains for the SMF's and DC fibre needs to be adjusted in order to achieve the appropriate Q-Factor. We have found out that the transmitted RF signal will be degraded after a certain distance by repeated amplifications, because of the added noise to the link due to an amplified spontaneous emission (ASE) [31]. Taking into consideration all practical aspect of the existing link structure, the use of NRZ and RZ modulation schemes enables us to come to the conclusion that RZ is the best choice for achieving the longest distance for the aforementioned bitrate. The proposed RF over fibre configuration schemes would have great impact on reducing the cost, reducing the system's complexity and avoiding usage of expensive devices and systems in order to achieve high data rate transmission over existing fibre optic systems.

\section{References:}

[1] T. Nagatsuma, and Y. Kado, "Microwave photonic devices and their applications to communications and Measurements", PIERS Online, vol.4, no. 3, (2008) pp.376-380

[2] R. Essiambre, R. W. Tkach, "Capacity Trends and Limits of Optical Communication Networks", Proceedings of the IEEE, vol. 100, no. 5, (2012) pp.1035-1055.

[3] B. Mani, A. Sivasubramanian, "Design of Symmetric dispersion compensated, long haul, single and multichannel optical Lightwave systems in telecommunications", IOSR-JECE, vol. 7, Iss. 4, (2013), pp. 69-82.

[4] T. Xie, M. Asif, H. Ali and H. M. R. Afzal, "Reparation of chromatic dispersion using dispersion compensation bank and bit-error rate analysis at various power levels in 40 Gbps fibre optics system", Image and Signal Processing (CISP), International Congress, (2014) pp.1058-1062.

[5] H. Ademgil, S. Haxha, "Bending Insensitive Large Mode Area Photonic Crystal Fibre”, Journal of Optics, vol. 122, (2011), pp.1950-1956.

[6] E. K. Akowuah, H. Ademgil, S. Haxha, F. A. Malek, "An Endlessly Single-Mode Photonic Crystal Fibre with Low Chromatic Dispersion, and Bend and Rotational Insensitivity" IEEE Journal of Lightwave Technology, vol. 27, no. 17, (2009), pp. 3940-3947.

[7] S. Akihide, M. Yutaka, "Performance evaluation of prechirped RZ and CS-RZ formats in high-speed transmission systems with dispersion management", Journal of Lightwave Technology, vol. 19, no. 12, (2001), pp. 1864-1871.

[8] A. Sousa, P. S. Andre, "Online Group-Velocity Dispersion Monitor Based on Clock Frequency Power Analysis", IEEE Photonics Technology Letters, vol. 24, no. 17, (2012), pp. 1533-1535.

[9] M. Asif, H. Ali, H. M. R. Afzal, T. A. Siddiqui, "Comparative analysis and power optimization for return to zero and non-return to zero coded transmission in $40 \mathrm{Gbit} / \mathrm{s}$ fibre optics system", Image and Signal Processing, 7th International Congress, (2014), pp.1022-1026.

[10] A. Cauvin, Y. Frignac, S. Bigo, "Nonlinear impairments at various bit rates in singlechannel dispersion-managed systems", Electronics Letters, vol. 39, no. 23, (2003), pp. 1670 .

[11]K. Miziya, S. K. Sudheer, A. C. Kuriakose, "Characterization of an optical communication system utilizing dispersion compensating fibre and nonlinear optical effects", Computing, Communications and Networking Technologies (ICCCNT), Fourth International Conference, (2013), pp. 1-6.

[12] N. I. Eappen, A. Sangeetha, "Analysis and performance comparison of inverse dispersion compensation technique for standard optical fibres," Computational Systems and Communications (ICCSC), First International Conference, (2014), pp. 364-369.

[13] S. Gupta, N. K. Shukla, S. Jaiswal, "Pre-, post, symmetric1 and 2 compensation techniques with RZ modulation", Recent Advances in Information Technology (RAIT), $1^{\text {st }}$ International Conference, (2012), pp. 251-255.

[14]M. I. Hayee, A. E. Willner, "Pre- and Post-Compensation of Dispersion and Nonlinearities in 10-Gb/s WDM systems", IEEE Photonics Technology Letters, vol. 9 , no. 9, (1997), pp. 1271-1273. 
[15] Y. A. Yaroshenko," Dispersion compensation with use of DCF fibre", Microwave and Telecommunication Technology (CriMiCo), 20 $0^{\text {th }}$ International Crimean Conference, (2010), pp. 362-363.

[16] R. S. Kaler, A. K. Sharma, T. S. Kamal, "Comparison of pre-, post and symmetric dispersion schemes for $10 \mathrm{Git} / \mathrm{s}$ NRZ links using standard and dispersion compensated fibres", Elsevier Optical Communication vol. 209, (2002), pp. 107-123.

[17]] S. Gupta, N. K. Shukla, S. Jaiswal, "Pre-, post, symmetric1 and 2 compensation techniques with RZ modulation", Recent Advances in Information Technology (RAIT), $1^{\text {st }}$ International Conference, (2012), pp. 251-255.

[18] D. Breuer, F. Küppers, A. Mattheus, E. G. Shapiro, I. Gabitov, and S. K. Turitsyn, "Symmetrical dispersion compensation for standard monomode-fiber-based communication systems with large amplifier spacing," Opt. Lett. 22, (1997), pp. 982984.

[19] L. Grüner-Nielsen, S. N. Knudsen, B. Edvold, T. Veng, D. Magnussen, C. C. Larsen, H. Damsgaard, "Dispersion Compensating Fibers", Optical Fiber Technology, Vol. 6, Iss. 2, (2000), pp 164-180.

[20] S. Haxha, H. Ademgil "Novel Design of Photonic Crystal Fibres with Low Confinement Losses, Nearly Zero Ultra-Flatted Chromatic Dispersion, Negative Chromatic Dispersion and Improved Effective Mode Area' Journal of Optics Communications, vol. 281, (2008), pp. 278-286.

[21] H. Ademgil, S. Haxha "Highly Birefringent Photonic Crystal Fibres with Ultra-Low Chromatic Dispersion and Low Confinement Losses", IEEE/OSA Journal of Lightwave Technology, vol. 26, no. 4, (2008), pp. 441-448.

[22] M. Joindot, M. Digonnet," Optical Transmission", Handbook of Optoelectronics, CRC Press, imprint of Taylor \& Francis Group, vol. 1, (2006), pp. 765 -795.

[23] W. Astar, "All-Optical Format Conversion of NRZ-OOK to RZ-OOK in a Silicon Nanowire Utilizing Either XPM or FWM and Resulting in a Receiver Sensitivity Gain of 2.5dB", IEEE Journal of Selected Topic in Quantum Electronics, vol. 16, no. 1, (2010), pp. 234-249.

[24] W. Jia, C. K. Chan, "Generation of Return-to-Zero Optical Pulses Using Directly Modulated Chirp Managed Laser", IEEE Photonics Technology Letters, vol. 24, no. 14, (2012), pp. 1227-1229.

[25] http://optiwave.com/

[26] http://www.vpiphotonics.com/index.php

[27] S. S. A. Khan, M. S. Islam, "Chromatic dispersion compensation using linearly chirped apodized fibre Bragg grating, "Electrical and Computer Engineering (ICECE), International Conference, (2010),pp. 9-12.

[28] E. Tipsuwannakul, J. Li, T. A. Eriksson, L. Egnell, F. Sjostrom, J. Pejnefors, P. A. Andrekson, M. Karlsson, "Influence of fibre-Bragg grating-induced group-delay ripple in high-speed transmission systems", IEEE/OSA Journal of Optical Communications and Networking, vol. 4, no. 6, (2012), pp. 514-521.

[29] C. Weber, C. Bunge, K. Petermann, "Fibre Nonlinearities in Systems Using Electronic Predistortion of Dispersion at 10 and 40 Gbit/s", Journal of Lightwave Technology, vol. 27, no. 16, (2009), pp. 3654-3661.

[30] R. J. Hoss, "Fibre Optic Communications Design Handbook', Prentice-Hall, Int- Ed, CRC PRESS, (1990), pp. 142-143.

[31] J. Kaur, N. Sharma, "Effects of Amplified Spontaneous Emission (ASE) on NRZ, RZ and CSRZ modulation formats in single channel light-wave system", Emerging Trends in Networks and Computer Communications (ETNCC), International Conference, (2011), pp. 61-64.

[32] G. P. Agrawal, "Applications of Nonlinear Fibre Optics", OPTICS AND PHOTONICS, Academic Press, vol. 1, no. 7, (2001), pp. 323-326.

[33] M. Wandel, P. Kristensen "Fibre designs for high figure of merit and high slope dispersion compensating fibre" Originally published J. Opt. Fibre, vol. 3, (2005), pp. 9-11. 
[34]A. Walter, G. S. Schaefer, "Chromatic dispersion variations in ultra-long-haul transmission systems arising from seasonal soil temperature variations", Optical Fibre Communication Conference and Exhibit, OFC, (2002), pp. 332-333.

[35] L. Gruner-Nielsen, M. Wandel, P. Kristensen, C. Jorgensen, L. V. Jorgensen, B. Edvold, B. Palsdottir, D. Jakobsen, "Dispersion-compensating fibres", Journal of Light Wave Technology, vil. 23, no. 11, (2005), pp. 3566-3579.

[36] Y. Wang, Y. Guan, "Performance simulations for a high-speed optical transmission system based on Optisystem," Image and Signal Processing (CISP), $7^{\text {th }}$ International Congress, (2014), pp. 907-911.

[37] M. Hirano, S. Hagihara, F. Ohkubo, Y. Koyano, T. Sasaki, "DCF module with low insertion loss, small residual dispersion, and low PMD," Optical Fibre Communication - includes post deadline papers (OFC), Conference on, CA, (2009), pp. 1-3.

[38] H. Ademgil, S. Haxha, "Endlessly Single Mode Photonic Crystal Fibre with Improved Effective Mode Area”, Journal Optics Communications, vol. 285, (2012), pp. 15141518.

[39] W. Freude, R. Schmogrow, B. Nebendahl, M. Winter, A. Josten, D. Hillerkuss, S. Koenig, J. Meyer, M. Dreschmann, M. Huebner, C. Koos, J. Becker, J. Leuthold, "Quality metrics for optical signals: Eye diagram, Q-factor, OSNR, EVM and BER," Transparent Optical Networks (ICTON), $14^{\text {th }}$ International Conference, (2012), pp. 1-4.

[40] M. Johnson, "Optical fibres, cables and systems", ITU-T Manual, (2009), pp. 141-143.

[41] K. Hagimoto, Y. Miyamoto, Y. Yamabayashi, "40-Gbit/s transmission systems," Optical Fibre Communication Conference and Exhibit, OFC '98 Technical Digest, (1998), pp. 114-115.

[42] S. Gringeri, E. B. Basch, T. J. Xia, “ Technical consideration for supporting data rate beyond 100 Gbit/s”, IEEE Communications Magazine, vol. 50, no. 2, (2012), pp. 21 30 .

[43] M. N. Zervas, R. I. Laming, D. N. Payne, "Efficient Erbium-Doped Fibre Amplifiers incorporating an Optical Isolator", Quantum Electronics, IEEE Journal, vol. 31, no. 3, (1995), pp. 472-480.

Acknowledgement: The authors would like to thank Dr Ian Flint and Dr Dalley James, from Selex ES-Finmeccanica UK industry, for their useful discussions on RF over fibre transmission systems. 